\title{
Some Special Classes of Semirings
}

\author{
C.Venkata Lakshmi \\ Dept. of Applied Mathematics \\ Sri Padmavati Mahila Visvavidyalayam \\ Tirupati, Andhra Pradesh, India.
}

\author{
T.Vasanthi \\ Dept. of Applied Mathematics \\ Yogi Vemana University, Kadapa - 516003 \\ Andhra Pradesh, India.
}

\begin{abstract}
In this paper, we discuss the structure of some special classes of semirings . Here the properties of Viterbi semirings; PRD semirings satisfying the identity $a b+a=a$, for all $a, b$ in $S$ and semirings satisfying the identity $\quad a+a b+a=a$, for all $\mathrm{a}, \mathrm{b}$ in $\mathrm{S}$ are studied. We proved that $(\mathrm{S},+, \cdot)$ is a semiring satisfying the identity $a+a b+a=a$, in which if $S$ contains the multiplicative identity which is also an additive identity, then $\mathrm{S}$ is a multiplicatively subidempotent semiring.
\end{abstract}

Keywords: Band; Left (Right) singular; PRD; Viterbi semiring; multiplicatively subidempotent; Weak commutative;

\section{Mathematics Subject Classification: 20M10, 16Y60.}

\section{INTRODUCTION}

In recent years interest in the study of partially ordered and fully ordered semigroups, groups, semirings, semimodules, rings and fields has been increasing enormously. Much of the theory of rings applied to arbitrary semirings. Some mathematicians go so far as to say that semirings are really the more fundamental concept, and specializing to rings should be seen in the same light as specializing to say algebra over the complex numbers.

The concept of semiring was first introduced by Vandiver in 1934. However the developments of the theory in semirings have been taking place since 1950 .The theory of semirings is attracting the attention of several algebraists due to its applications to Computer Science, Optimization theory, Automata theory, Formal language theory and the Mathematical Modeling of Quantum Physics. Especially semirings with different properties have become important in theoretical computer science.

The semiring identities are taken from the book of Jonathan S.Golan[2], entitled "Semirings and their Applications".

In this paper, we investigate the additive and multiplicative properties of some special classes of Semirings.

\section{Definition 1.1}

A triple $(S,+, \cdot)$ is said to be a semiring if $S$ is a non - empty set and ",$+ \bullet$ " are binary operations on $\mathrm{S}$ satisfying that

(i) $(\mathrm{S},+)$ is a semigroup

(ii) $(\mathrm{S}, \bullet)$ is a semigroup

(iii) $\mathrm{a}(\mathrm{b}+\mathrm{c})=\mathrm{ab}+\mathrm{ac}$ and $(\mathrm{b}+\mathrm{c}) \mathrm{a}=\mathrm{b} a+\mathrm{ca}$, for all $\mathrm{a}, \mathrm{b}, \mathrm{c}$ in $\mathrm{S}$.

Example of Semiring 1.2

(i) The set of natural numbers under the usual addition, multiplication (ii) Any ring $(\mathrm{R},+, \bullet)$.

(iii) Every distributive lattice $(L, \wedge, \vee)$.

\section{Definition 1.3}

A semigroup $(\mathrm{S},+)$ is said to be

(i) left singular, if it satisfies the identity $a+b=a$, for all $a, b$ in $S$;

(ii) right singular, if it satisfies the identity $a+b=b$, for all $a, b$ in $S$;

(iii) singular, if it is both left as well as right singular.

\section{Definition 1.4:}

A semigroup $(\mathrm{S}, \bullet)$ is said to be

(i) left singular, if it satisfies the identity $a b=a$, for all a, $\mathrm{b}$ in $\mathrm{S}$;

(ii) right singular, if it satisfies the identity $\mathrm{ab}=\mathrm{b}$, for all $\mathrm{a}, \mathrm{b}$ in $\mathrm{S}$;

(iii) singular, if it is both left as well as right singular.

\section{Definition 1.5}

An element 'a' of a semiring $S$ is multiplicatively subidempotent if and only if $\mathrm{a}+\mathrm{a}^{2}=\mathrm{a}$ and $\mathrm{S}$ is multiplicatively subidempotent if and only if each of its elements is multiplicatively subidempotent.

\section{Definition 1.6}

A viterbi semiring is a semiring in which $\mathrm{S}$ is additively idempotent and multiplicatively subidempotent i.e., $a+a=a$ and $a+a^{2}=a$, for all ' $a$ ' in $S$.

The concept of viterbi Semiring is taken from the book of Jonathan S.Golan [3], entitled "Semirings and Affine Equations over Them: Theory and Applications".

Theorem 1.7: Every semiring $(\mathrm{S},+, \bullet)$ in which $(\mathrm{S},+)$ and $(\mathrm{S}$, -) are left singular semigroups, then $(\mathrm{S},+, \cdot)$ is a viterbi semiring.

Proof: By hypothesis, (S, +) and (S, •) are left singular semigroups

i.e. $a+b=a$ and $a b=a$, for all $a, b$ in $S$

Consider $\mathrm{a}+\mathrm{b}=\mathrm{a}$

Taking $\mathrm{b}=\mathrm{a}$

$\Rightarrow \mathrm{a}+\mathrm{a}=\mathrm{a} \rightarrow(1)$

And $\mathrm{ab}=\mathrm{a}$

Taking $\mathrm{b}=\mathrm{a}$

$\Rightarrow \mathrm{a} . \mathrm{a}=\mathrm{a}$

$\Rightarrow \mathrm{a}^{2}=\mathrm{a}$

$\Rightarrow \mathrm{a}+\mathrm{a}^{2}=\mathrm{a}+\mathrm{a}$

$\Rightarrow \mathrm{a}+\mathrm{a}^{2}=\mathrm{a} \rightarrow(2) \quad(\because$ from $(1), \mathrm{a}+\mathrm{a}=\mathrm{a})$

$\therefore$ From (1) \& (2), $(\mathrm{S},+, \cdot)$ is a viterbi semiring.

Remark: Converse of theorem 1.7, is not necessarily true. This is evident from the following example 


\section{Example 1.8:}

\begin{tabular}{|c|c|c|}
\hline+ & $\mathbf{a}$ & $\mathbf{b}$ \\
\hline $\mathbf{a}$ & $\mathbf{a}$ & $\mathbf{a}$ \\
\hline $\mathbf{b}$ & $\mathbf{b}$ & $\mathbf{b}$ \\
\hline
\end{tabular}

\begin{tabular}{|c|c|c|}
\hline$\cdot$ & $\mathbf{a}$ & $\mathbf{b}$ \\
\hline $\mathbf{a}$ & $\mathbf{a}$ & $\mathbf{a}$ \\
\hline $\mathbf{b}$ & $\mathbf{b}$ & $\mathbf{b}$ \\
\hline
\end{tabular}

Definition 1.9

An element $x$ in a semigroup $(S,+)$ is said to be an absorbing if $x+a=a+x=x$, for every ' $a$ ' in $S$.

\section{Definition: 1.10}

An element ' $\mathrm{x}$ ' in a semiring $(\mathrm{S},+, \cdot)$ is said to be

(i) an additive identity if $\mathrm{a}+\mathrm{x}=\mathrm{x}+\mathrm{a}=\mathrm{a}$.

(ii) a multiplicative identity if $\mathrm{ax}=\mathrm{xa}=\mathrm{a}$.

(iii) an additive zero if $\mathrm{a}+\mathrm{x}=\mathrm{x}+\mathrm{a}=\mathrm{x}$ and

(iv) a multiplicative zero if $\mathrm{ax}=\mathrm{xa}=\mathrm{x}$, for every ' $\mathrm{a}$ ' in $\mathrm{S}$.

\section{Note}

(1) When we say a semiring with zero (or) a t.o.s.r. with zero mean multiplicative zero.

(2) Let ' $x$ ' is said to be left(right) additive identity if $x$ $+a=a(a+x=a)$ for every ' $a$ ' in S.

(3) Let ' $x$ ' is said to be left (right) additive zero if $x+a=x$ (a $+x=x)$ for every ' $a$ ' in S.

Theorem 1.11: Let $(\mathrm{S},+, \cdot)$ be a semiring containing multiplicative identity 1 . Then

(i) If 1 is an absorbing element w.r.to ' + ', then $(\mathrm{S},+, \bullet)$ is a viterbi semiring

(ii) The converse of (i) is also true if $(\mathrm{S}, \bullet)$ is left cancellative and $(\mathrm{S},+)$ is commutative

Proof: By hypothesis, S contains multiplicative identity 1

(i) Taking 1 as an absorging element w.r.to '+'

Consider $1+\mathrm{a}=1$, for all ' $\mathrm{a}$ ' in $\mathrm{S}$

$\Rightarrow \mathrm{a}(1+\mathrm{a})=\mathrm{a} .1$

$\Rightarrow \mathrm{a}+\mathrm{a}^{2}=\mathrm{a} \rightarrow(1)$

Also $1+1=1$

$\Rightarrow \mathrm{a}(1+1)=\mathrm{a} .1$

$\Rightarrow \mathrm{a}+\mathrm{a}=\mathrm{a} \quad \rightarrow(2)$

$\therefore$ From (1) \& (2), $(\mathrm{S},+, \cdot)$ is a viterbi semiring.

(ii) Consider $a+a^{2}=a$, for all ' $a$ ' in $S$

$\Rightarrow \mathrm{a} .1+\mathrm{a}^{2}=\mathrm{a} .1$

$\Rightarrow \mathrm{a}(1+\mathrm{a})=\mathrm{a} \cdot 1$

$\Rightarrow 1+\mathrm{a}=1 \quad(\because(\mathrm{S}, \bullet)$ is left cancellative $)$

$\Rightarrow 1+\mathrm{a}=\mathrm{a}+1=1 \rightarrow(3)(\because(\mathrm{S},+)$ is commutative $)$

$\therefore$ From (3), 1 is an absorging element w.r.to ' + '.

Example 1.12: This is an example for theorem 1.11

\begin{tabular}{|c|c|c|}
\hline+ & 1 & $\mathbf{a}$ \\
\hline 1 & 1 & 1 \\
\hline $\mathbf{a}$ & $\mathbf{1}$ & $\mathbf{a}$ \\
\hline
\end{tabular}

\begin{tabular}{|l|l|l|}
\hline$\bullet$ & $\mathbf{1}$ & $\mathbf{a}$ \\
\hline $\mathbf{1}$ & $\mathbf{1}$ & $\mathbf{a}$ \\
\hline $\mathbf{a}$ & $\mathbf{a}$ & $\mathbf{a}$ \\
\hline
\end{tabular}

Theorem 1.13: Let $(\mathrm{S},+, \cdot)$ be a semiring satisfying the identity $a+a b+a=a$, for all $a, b$ in $S$. If $S$ contains the multiplicative identity which is also an additive identity, then $\mathrm{S}$ is a multiplicatively subidempotent semiring.

Proof: Since $a+a b+a=a$, for all $a, b$ in $S$

Let 'e' be the multiplicative identity is also an additive identity

i.e. $a \cdot e=e \cdot a=a \& a+e=e+a=a$
Given $\mathrm{a}+\mathrm{ab}+\mathrm{a}=\mathrm{a}$, for all $\mathrm{a}, \mathrm{b}$ in $\mathrm{S}$

Taking $\mathrm{b}=\mathrm{a}$

$\Rightarrow a+a^{2}+a=a$

$\Rightarrow \mathrm{a}+\mathrm{a}[\mathrm{a}+\mathrm{e}]=\mathrm{a}$

$\Rightarrow \mathrm{a}+\mathrm{a}^{2}=\mathrm{a}+0$

$\Rightarrow a+a^{2}=a$, for all ' $a$ ' in $S$

Hence $\mathrm{S}$ is multiplicatively subidempotent semiring.

\section{Definition 1.14}

An element ' $\mathrm{x}$ ' in a semigroup $(\mathrm{S},+)$ is said to be an additive idempotent if $\mathrm{x}+\mathrm{x}=\mathrm{x}$.

A semigroup $(\mathrm{S},+)$ is said to be a band if every element in $\mathrm{S}$ is an idempotent.

\section{Note}

$\mathrm{E}(+)$ denotes the set of all additive idempotents in $(\mathrm{S},+)$

$|\mathrm{E}(+)|$ denotes the cardinal number of the set $\mathrm{E}[+]$.

\section{Definition 1.15}

A semiring $(\mathrm{S},+, \cdot)$ is said to be an additively idempotent semiring if $(\mathrm{S},+)$ is a band. i.e., $a=a+a$, for every ' $a$ ' in $S$.

\section{Definition 1.16}

An element $\mathrm{x}$ in a semigroup $(\mathrm{S}, \bullet)$ is said to be multiplicative idempotent if $\mathrm{x}^{2}=\mathrm{x}$.

A semigroup $(S, \bullet)$ is said to be a band if every element in $S$ is an idempotent.

Note

$\mathrm{E}(\bullet)$ denotes the set of all multiplicative idempotents in $\bullet$.

$|\mathrm{E}(\bullet)|$ denotes the cardinal number of the set $\mathrm{E}[\bullet]$.

\section{Definition 1.17}

A semiring $(\mathrm{S},+, \bullet)$ is said to be multiplicatively idempotent semiring if $(\mathrm{S}, \bullet)$ is a band.

$$
\text { i.e., } a=a^{2} \text {, for every ' } a \text { ' in } S \text {. }
$$

\section{Definition 1.18}

A semigroup $(\mathrm{S}, \bullet)$ is said to satisfy weak commutative if abc $=\mathrm{bac}$, for all $\mathrm{a}, \mathrm{b}, \mathrm{c}$ in $\mathrm{S}$.

Theorem 1.19: Let $(S,+, \bullet)$ be a multiplicatively idempotent semiring. If $S$ satisfying the identity $a+a b+a=a$ for all $\mathrm{a}, \mathrm{b}$ in $\mathrm{S}$ then $\mathrm{S}$ is additively idempotent and in addition $\mathrm{S}$ satisfies the condition that $\mathrm{ab}+\mathrm{ba}=\mathrm{ab}$, for all $\mathrm{a}, \mathrm{b}$ in $\mathrm{S}$, if $(\mathrm{S}, \bullet)$ is weak commutative.

Proof: Let a $\in \mathrm{S}$

Consider $a+a=(a+a)^{2}$, for all $a$, in $S$

$(\because(\mathrm{S},+, \bullet)$ is a multiplicatively idempotent semiring)

$\Rightarrow \mathrm{a}+\mathrm{a}=(\mathrm{a}+\mathrm{a})(\mathrm{a}+\mathrm{a})$

$\Rightarrow a+a=a^{2}+a^{2}+a^{2}+a^{2}$

$\Rightarrow a+a=a^{2}+a(a+a)+a^{2}$

$\Rightarrow a+a=a+a(a+a)+a$

$(\because(\mathrm{S},+, \bullet)$ is a multiplicatively idempotent semiring)

$\Rightarrow \mathrm{a}+\mathrm{a}=\mathrm{a} \quad(\because \mathrm{a}+\mathrm{ab}+\mathrm{a}=\mathrm{a})$

$\therefore \mathrm{S}$ is additively idempotent.

Moreover, let $\mathrm{a}, \mathrm{b} \in \mathrm{S}$

$$
\begin{aligned}
\Rightarrow a b+b a & =a b+b a+a b \quad(\because b a=b a+a b) \\
& =(a b+b a+a b)^{2} \\
& =(a b+b a+a b)(a b+b a+a b) \\
& =a b(a b+b a+a b)+b a(a b+b a+a b)+a b(a b+b a+a b)
\end{aligned}
$$




$$
\begin{aligned}
& \Rightarrow a b+b a=a b(a b+b a+a b)+b\left(a^{2} b+a b a+a^{2} b\right)+a b(a b+b a+a b) \\
& \quad=(a b+a b a+a b)+b(a b+a b a+a b)+(a b+a b a+a b) \\
& (\because S \text { is multiplicatively idempotent semiring }) \\
& \Rightarrow a b+b a=a b+b(a b)+a b(\because a+a b+a=a) \\
& \quad=a b+a b(b)+a b \\
& (\because(S, \cdot) \text { is weak commutative, } b a b=a b b) \\
& \Rightarrow a b+b a=a b \quad(\because a+a b+a=a) \\
& \begin{aligned}
\therefore a b+b a=a b, \text { for all } a, b \text { in } S .
\end{aligned}
\end{aligned}
$$

\section{Definition 1.20}

A semiring $(\mathrm{S},+, \bullet)$ is said to be a Positive Rational Domain $(\mathrm{PRD})$ if and only if $(\mathrm{S}, \bullet)$ is an abelian group.

Theorem 1.21: Let $(S,+, \bullet)$ be a PRD semiring satisfying the identity $\mathrm{ab}+\mathrm{a}=\mathrm{a}$, for all $\mathrm{a}, \mathrm{b}$ in $\mathrm{S}$. Then the following are true.

(i) $\mathrm{a}+\mathrm{ab}=\mathrm{b}+\mathrm{ab}$, for all $\mathrm{a}, \mathrm{b}$ in $\mathrm{S}$

(ii) $a+a^{2}=a^{2}$ and $a^{2}+a=a$, for all ' $a$ ' in $S$

Proof: By hypothesis, $a b+a=a$, for all $a, b$ in $S$

$\Rightarrow \mathrm{a} \mathrm{a}^{-1}+\mathrm{a}=\mathrm{a}$, for all $\mathrm{a}, \mathrm{a}^{-1}$ in $\mathrm{S}$

( $\because \mathrm{S}$ is a PRD semiring)

$\Rightarrow 1+\mathrm{a}=\mathrm{a}$

$\therefore 1+\mathrm{a}=\mathrm{a}$, for all 'a' in $\mathrm{S} \rightarrow(1)$

(i) $\mathrm{ab}=\mathrm{a}(1+\mathrm{b})(\because 1+\mathrm{b}=\mathrm{b})$

$$
=\mathrm{a}+\mathrm{ab}
$$

Also $a b=(1+a) b(\because 1+a=a)$

$$
\begin{gathered}
=b+a b \\
\therefore a+a b=b+a b
\end{gathered}
$$

(ii) $1+\mathrm{a}=\mathrm{a}$, for all $\mathrm{a}$ in $\mathrm{S}$

$\Rightarrow \mathrm{a}(1+\mathrm{a})=\mathrm{a} \cdot \mathrm{a}$

$\Rightarrow \mathrm{a}+\mathrm{a}^{2}=\mathrm{a}^{2}$

Now $a b+a=a$, for all $a, b$ in $S$

Taking $\mathrm{b}=\mathrm{a}$

$\Rightarrow \mathrm{a} . \mathrm{a}+\mathrm{a}=\mathrm{a}$, for all ' $\mathrm{a}$ ' in $\mathrm{S}$

$\Rightarrow \mathrm{a}^{2}+\mathrm{a}=\mathrm{a}$

Hence $\mathrm{a}+\mathrm{a}^{2}=\mathrm{a}^{2}$ and $\mathrm{a}^{2}+\mathrm{a}=\mathrm{a}$, for all ' $\mathrm{a}$ ' in $\mathrm{S}$.

\section{CONCLUSION}

The studies of multiplicative structure of some special classes of Semirings containing the multiplicative identity had the additive structure and vice versa.

\section{REFERENCES}

[1] Arif Kaya and M.Satyanarayana, "Semirings satisfying properties of distributive type", Proceeding of the American Mathematical Society, Volume 82, Number 3, July 1981.

[2] Jonathan S.Golan, "Semirings and their Applications".

[3] Jonathan S.Golan, "Semirings and Affine Equations over Them : Theory and Applications". Kluwer Academic. 